\title{
Supramolecular Velcro for Underwater Adhesion
}

Gluing wet surfaces or even surfaces under water is a challenge. In the journal Angewandte Chemie, Korean scientists have now introduced a completely new concept. They were able to achieve reversible underwater adhesion by using supramolecular "Velcro".

Dreviously, researchers have primarily attempted to mimic natural underwater "adhesives" like the adhesive molecules used by mussels. Such substances adhere well in wet surroundings, but the adhesion is not reversible. A team led by Kimoon Kim at Pohang University of Science and Technology in Korea uses socalled host-guest interactions between water-soluble host molecules with a hydrophobic pocket and ionic guest molecule with a hydrophobic block, which form robust noncovalent bonds in water. The repulsion of water molecules is the driving force for formation of the bond.

The researchers chose curcurbit [7] uril as their host and aminomethylferrocene as the guest. Die Cucurbit[n]uril (n $=5,6,7,8$ and 10) are macrocycles - large, ring-shaped molecules made of multiple glycoluril units that can bind other molecules within their cavities. Their name is derived from cucurbita, the Latin name for pumpkin, which they resemble in shape. Ferrocenes are also known as sandwich compounds. The "bread" consists of two aromatic rings of five carbon atoms, while the "filling" is a single iron atom held in the middle. The ferrocene chosen by the researchers lodges very
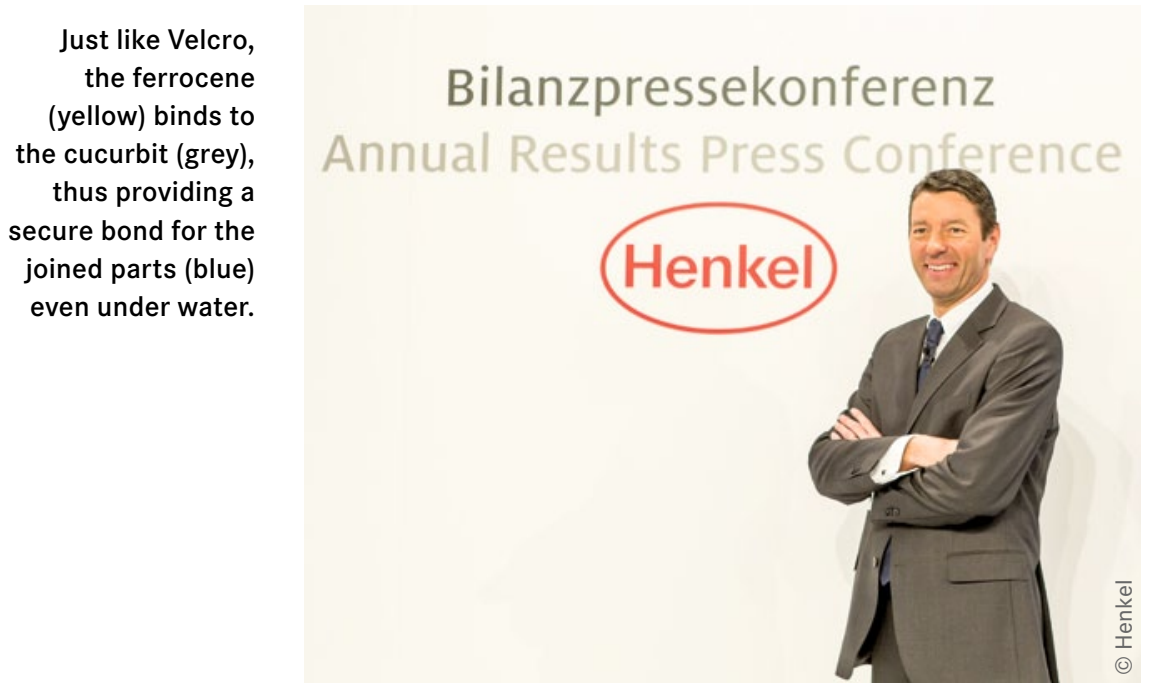

securely and specifically inside a "pumpkin" made of seven glycoluril units.

The researchers produced some silicon strips with many "pumpkins" attached, as well as some with many "sandwiches". When these strips come into contact with each other they stick together tightly, similar to Velcro. Once stuck together, a $1 \times 1 \mathrm{~cm}$ piece of this supramolecular Velcro can hold a weight of $2 \mathrm{~kg}$ in water. After drying in air it can hold as much as $4 \mathrm{~kg}$.

Like a macroscopic Velcro strip, the molecular version can be separated with a strong pull and reused multiple times. The adhesion can also be reversed chemically through application of a hypochlorite solution, which oxidizes the iron atoms. After reduction with an agent such as ascorbic acid, the Velcro can adhere again. Because the materials used are biocompatible, biological applications may be possible, for example in surgery suture or repairing live tissue. Mussel mimetic underwater adhesives are not suitable here, as they require strong oxidizing agents for curing.

\section{BASF and Markor Plan Joint Ventures in China}

$\mathrm{B}$ ASF and Xinjiang Markor Chemical Industry Co., Ltd. (Markor) plan to establish two joint ventures for the production of butanediol (BDO) and polytetrahydrofuran (polytetramethylene ether glycol ) in Korla, Xinjiang Uygur autonomous region, Northwest China. The joint venture agreements have already been signed but are subject to further closing conditions and regulatory approv- als. The joint venture companies intend to build a plant for the production of BDO and one for polytetrahydrofuran, which are both planned to go on stream in 2015. The annual capacities of the plants located in Korla will be 100,000 tons of BDO and 50,000 tons of polytetrahydrofuran.

Polytetrahydrofuran is primarily used to make elastic spandex fi- bres for a large variety of textiles, but it also serves as a chemical building block for thermoplastic polyurethanes (TPU). Other applications include thermoplastic polyetheresters, polyetheramides and cast elastomers. BDO is used in the manufacturing of technical plastics, polyurethanes, solvents, electronic chemicals and elastic fibres. 